\title{
ABCG2 impairs the activity of the aurora kinase inhibitor tozasertib but not of alisertib
}

\author{
Martin Michaelis ${ }^{1,2+}$, Florian Selt ${ }^{1,4+}$, Florian Rothweiler ${ }^{1}$, Michael Wiese ${ }^{3}$ and Jindrich Cinatl Jr. ${ }^{1 *}$
}

\begin{abstract}
Background: Recently, we have shown that the ATP-binding cassette (ABC) transporter $A B C B 1$ interferes with the anti-cancer activity of the pan-aurora kinase inhibitor tozasertib (VX680, MK-0457) but not of the aurora kinase $A$ and $B$ inhibitor alisertib (MLN8237). Preliminary data had suggested tozasertib also to be a substrate of the ABC transporter $A B C G 2$, another $A B C$ transporter potentially involved in cancer cell drug resistance. Here, we studied the effect of ABCG2 on the activity of tozasertib and alisertib.

Results: The tozasertib concentration that reduces cell viability by $50 \%\left(I_{50}\right)$ was dramatically increased in $A B C G 2-$ transduced UKF-NB-3 ${ }^{A B C G 2}$ cells (48.8-fold) compared to UKF-NB-3 cells and vector-transduced control cells. The ABCG2 inhibitor WK-X-34 reduced tozasertib IC ${ }_{50}$ to the level of non-ABCG2-expressing UKF-NB-3 cells. Furthermore, $A B C G 2$ depletion from UKF-NB-3 ${ }^{A B C G 2}$ cells using another lentiviral vector expressing an shRNA against the bicistronic mRNA of ABCG2 and eGFP largely re-sensitised these cells to tozasertib. In contrast, alisertib activity was not affected by ABCG2 expression.
\end{abstract}

Conclusions: Tozasertib but not alisertib activity is affected by ABCG2 expression. This should be considered within the design and analysis of experiments and clinical trials investigating these compounds.

Keywords: ABCG2, Drug resistance, Aurora kinase inhibitor, Tozasertib, VX680, MK-0457, Alisertib, MLN8237

\section{Background}

The aurora kinases $\mathrm{A}, \mathrm{B}$, and $\mathrm{C}$ are involved in spindle apparatus organisation during cell division $[1,2]$. Inhibitors of aurora kinases represent a novel class of anticancer drugs currently under pre-clinical and clinical investigation [1-5]. Aurora kinases have been suggested to be potential drug targets in neuroblastoma [6-15], the most frequent extracranial solid childhood tumour. About half of neuroblastoma patients suffer from highrisk disease associated with overall survival rates below $50 \%$ despite intensive therapy $[16,17]$.

Recently, we showed that aurora kinases may represent targets in therapy-refractory neuroblastoma. In particular, p53 wild-type neuroblastoma cells were sensitive to aurora kinase inhibitors [15]. Notably, only a small

\footnotetext{
*Correspondence: Cinatl@em.uni-frankfurt.de

${ }^{\dagger}$ Martin Michaelis and Florian Selt have contributed equally to this work ${ }^{1}$ Institut für Medizinische Virologie, Klinikum der Goethe-Universität, Paul Ehrlich-Str. 40, 60596 Frankfurt Am Main, Germany

Full list of author information is available at the end of the article
}

fraction of neuroblastomas harbours p53-mutant cells $[18,19]$. In addition, we confirmed previous assumptions that $\mathrm{ABCB} 1$ expression confers resistance to the panaurora kinase inhibitor tozasertib (VX680, MK-0457) $[15,20,21]$. In contrast, the activity of the aurora kinase A and B inhibitor alisertib (MLN8237) was not affected by the presence of $\mathrm{ABCB} 1$ (also known as P-glycoprotein or MDR1) [15]. Tozasertib was suggested to also interfere with ABCG2 (also known as BCRP) [20], another ATPbinding cassette $(\mathrm{ABC})$ transporter known to be involved in cancer cell drug resistance [22], but conclusive experimental evidence has been missing. Moreover, there is no information on a possible interaction of alisertib with ABCG2 available in the public domain. Thus, we here investigated the effects of ABCG2 expression on the anticancer effects of tozasertib and alisertib.

\section{Methods \\ Drugs \\ Tozasertib and alisertib were purchased from Selleck Chemicals (Houston, TX, USA), mitoxantrone from}


Gry-Pharma GmbH (Kirchzarten, Germany). WK-X-34 was synthesised as described before [23].

\section{Cells}

The MYCN-amplified, ABCB1-negative neuroblastoma cell line UKF-NB-3 was derived from a bone marrow metastasis of a stage IV neuroblastoma patient [24] and propagated in IMDM supplemented with $10 \%$ FBS, $100 \mathrm{IU} / \mathrm{ml}$ penicillin and $100 \mathrm{mg} / \mathrm{ml}$ streptomycin at $37{ }^{\circ} \mathrm{C}$. Cells were routinely tested for mycoplasma contamination and authenticated by short tandem repeat profiling. Cells showing high expression of ABCG2 were established as described previously $[25,26]$ using the lentiviral gene ontology (LeGO) vector technology $[27,28]$ (http://www.LentiGO-Vectors.de).

\section{Viability assay}

Cell viability was tested by the 3-(4,5-dimethylthiazol2-yl)-2,5-diphenyltetrazolium bromide (MTT) dye reduction assay after $120 \mathrm{~h}$ incubation modified as described previously [29].

\section{ABCG2 depletion in UKF-NB-3 ${ }^{\text {ABCG2 }}$ cells}

The LeGO-iG2 vector that we used for the expression of ABCG2 (LeGO-iG2-ABCG2) is a bicistronic vector with an internal ribosome entry site (IRES) that links the expression of the fluorescent marker gene to the expression of another gene of interest (here ABCG2) [25-29]. Previously, it was shown that the expression of genes from this bicistronic vector can be depleted by the use of a second vector encoding an shRNA against eGFP [27]. Here, we cloned the eGFP-shRNA (GCACGACTTCTTCAAGTCC [27]) into the LeGO-X vector that uses dsRedExpress (orange emission, $584 \mathrm{~nm}$ ) as marker [27] (http://www.LentiGo-Vectors.de) resulting in the vector LeGO-X-GFP2.

\section{Flow cytometry}

An antibody directed against ABCG2 (Kamiya Biomedical Company, Seattle, WA, USA), followed by secondary antibody labelled with Phycoerythrin (R\&D, Wiesbaden, Germany) was used to detect protein expression by flow cytometry (FACSCanto, BD Biosciences, Heidelberg, Germany).

\section{Statistics}

Results are expressed as mean $\pm \mathrm{SD}$ of at least three experiments. Comparisons between two groups were performed using Student's $t$ test. Three and more groups were compared by ANOVA followed by the StudentNewman-Keuls test. P values lower than 0.05 were considered to be significant.

\section{Results}

Effects of tozasertib and alisertib on the viability of ABCG2-expressing cells

The concentration that reduces cell viability by $50 \%$ $\left(\mathrm{IC}_{50}\right)$ was dramatically increased in ABCG2-transduced UKF-NB-3 ${ }^{\text {ABCG2 }}$ cells for tozasertib (48.8-fold) and mitoxantrone (a cytotoxic ABCG2 substrate that was used as control, 296.5-fold) (Fig. 1; Additional file 1: Table S1). In the presence of the ABCG2 inhibitor WK-X34 , the tozasertib and mitoxantrone $\mathrm{IC}_{50}$ values were reduced to the level of non-ABCG2-expressing UKFNB-3 cells (Fig. 1; Additional file 1: Table S1). In contrast, alisertib activity was not affected by ABCG2 expression (Fig. 1; Additional file 1: Table S1).

\section{Effects of ABCG2 depletion on tozasertib efficacy}

In order to deplete ABCG2 from UKF-NB-3 ${ }^{\mathrm{ABCG} 2}$ cells, we additionally transduced these cells with the LeGO-XGFP2 vector encoding an shRNA directed against eGFP. Fluorescence microscopy indicated effective reduction

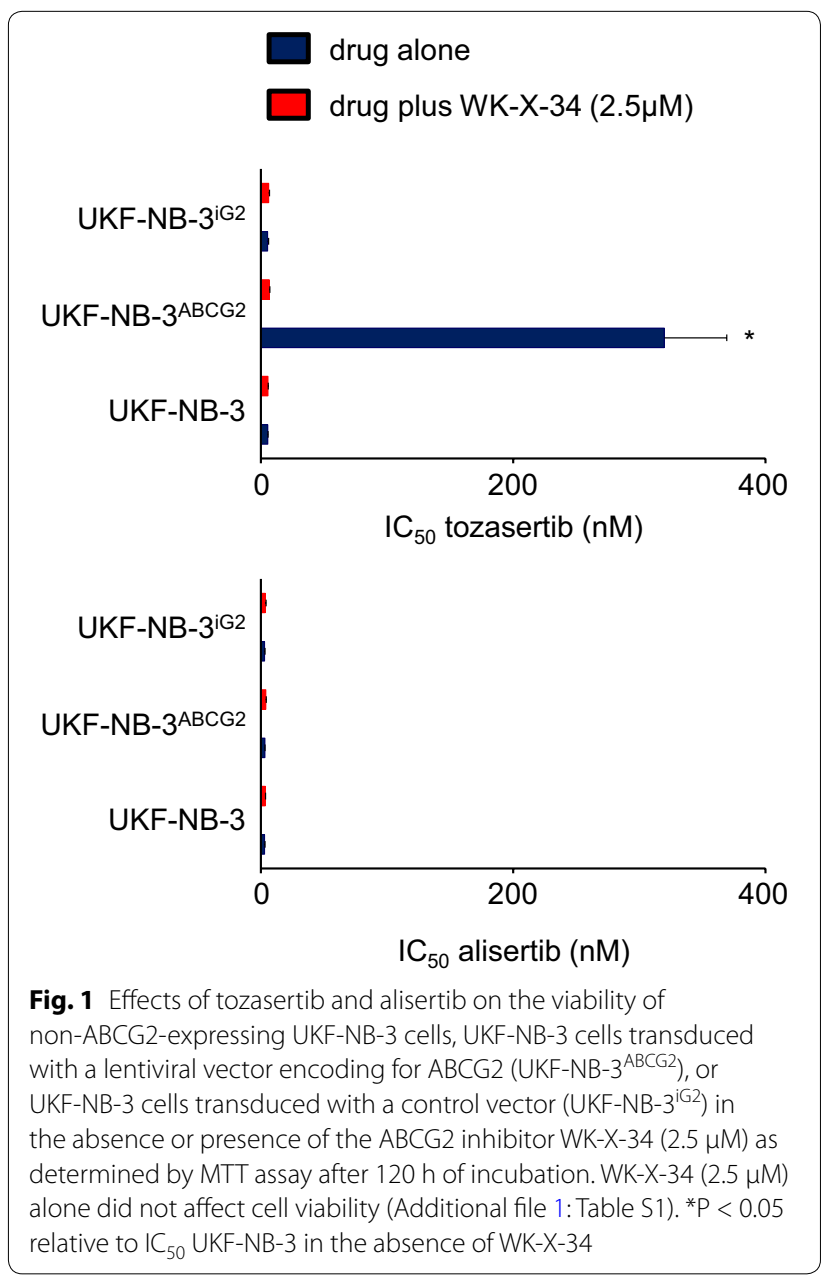


of eGFP protein levels in UKF-NB-3 ${ }^{\text {ABCG2-XGFP2 }}$ cells (Fig. 2a). Moreover, flow cytometric analysis demonstrated decreased ABCG2 levels in UKF-NB-3 ${ }^{\text {ABCG2-XGFP2 }}$ cells (Fig. 2b). In accordance with the results from the use of the ABCG2 inhibitor WK-X-34, UKF-NB-3 ${ }^{\text {ABCG2-XGFP2 }}$ cells were re-sensitised to tozasertib and the cytotoxic ABCG2 substrate mitoxantrone (Fig. 3).

\section{Discussion}

Knowledge about the interaction of drug candidates with $\mathrm{ABC}$ transporters is important for their investigation and (pre-)clinical development because ABC transporters are expressed at organ and tissue barriers determining drug body distribution [30]. Moreover, ABCG2 expression may be involved in cancer cell drug resistance [22].

Previously, we had shown that the pan aurora kinase inhibitor tozasertib that is a frequently used tool compound [with 128 articles in the Pubmed (http://www. ncbi.nlm.nih.gov/pubmed) as of 19th August 2015] but not the aurora kinase A and B inhibitor alisertib that substantially differs in structure from tozasertib and is under investigation in multiple clinical trials ([3-5], 50 clinical

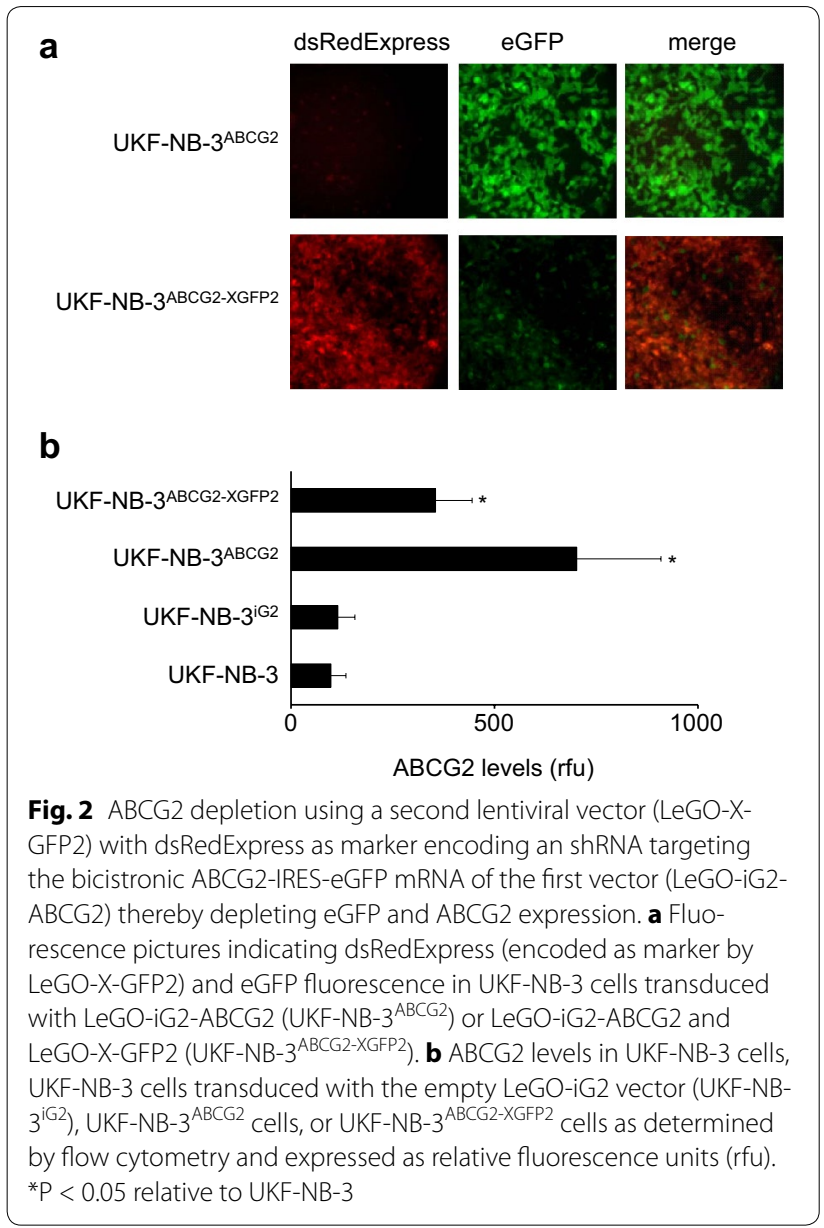

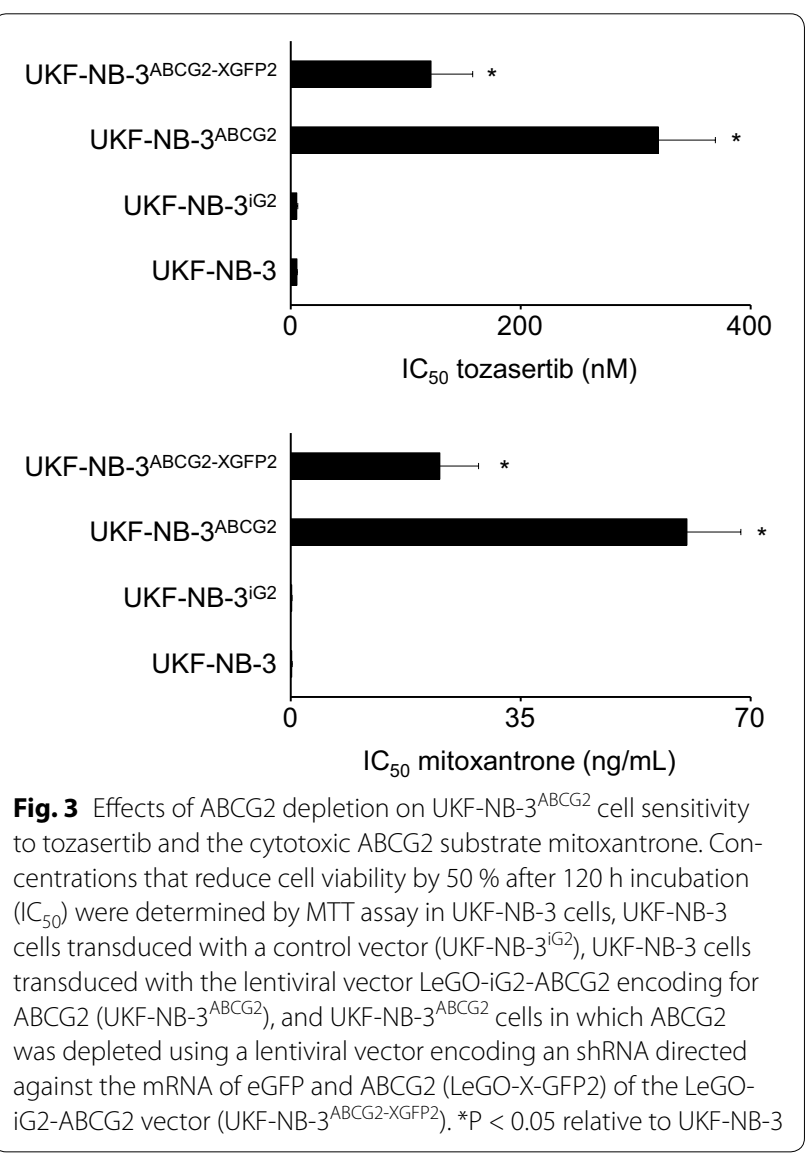

studies of alisertib are registered at http://www.clinicaltrials.gov as of 19th August 2015) interferes with ABCB1mediated drug transport [15]. Here, we provide evidence that the efficacy of tozasertib is also affected by ABCG2 expression. ABCG2 expression reduced cancer cell sensitivity to tozasertib and the cytotoxic ABCG2 substrate mitoxantrone. Interference with ABCG2 using WK-X-34, an ABCG2 inhibitor, or RNAi-mediated ABCG2 depletion resulted in re-sensitisation of ABCG2-expressing cells to tozasertib (and mitoxantrone). This is in concordance with previous findings suggesting an interaction of tozasertib with ABCG2 [20] although conclusive experimental evidence had been missing. Cancer cell lines adapted to the aurora kinase inhibitor AZD1152 had been shown to express high levels of ABCG2 and to be cross-resistant to tozasertib [20]. However, studies confirming that there is a functional relationship between high ABCG2 expression and decreased tozasertib sensitivity had not been performed. Moreover, this is the first study that investigated a potential effect of ABCG2 on the activity of alisertib and provides evidence that ABCG2 expression does not impair the efficacy of alisertib.

In conclusion, the differential effects of ABCG2 on tozasertib and alisertib activity should be carefully 
considered within the design and analysis of experiments and clinical trials investigating these compounds.

\section{Availability of supporting data}

The data sets supporting the results of this article are included within the article and its Additional file 1: Table S1.

\section{Additional file}

Additional file 1: Table S1. Effects of tozasertib, alisertib, and the cytotoxic ABCG2 substrate mitoxantrone on the viability of non- ABCG2expressing UKF-NB-3 cells, UKF-NB-3 cells transduced with a lentiviral vector encoding for ABCG2 (UKF-NB-3ABCG2), or UKF-NB-3 cells transduced with a control vector (UKF-NB-3piG2) in the absence or presence of the ABCG2 inhibitor WK-X-34.

\section{Abbreviations}

MTT: 3-(4,5-dimethylthiazol-2-yl)-2,5-diphenyltetrazolium bromide; ABC transporter: ATP-binding cassette transporter; $I C_{50}$ : concentration that reduces cell viability by $50 \%$; FBS: foetal bovine serum; IMDM: Iscove's modified Dulbecco's medium.

\section{Authors' contributions}

MM and JCjr designed the study, analysed and interpreted data, and wrote the manuscript. FS performed the MTT assays and the lentiviral transduction experiments, analysed and interpreted data. FR directed and performed flow cytometry experiments and analysed and interpreted data. MW provided WK-X-34 and participated in the study design. All authors read and approved the final manuscript.

\section{Author details \\ ${ }^{1}$ Institut für Medizinische Virologie, Klinikum der Goethe-Universität, Paul Ehrlich-Str. 40, 60596 Frankfurt Am Main, Germany. ${ }^{2}$ Present Address: Centre for Molecular Processing and School of Biosciences, University of Kent, Canterbury CT2 7NJ, UK. ${ }^{3}$ Pharmaceutical Institute, University of Bonn, An der Immenburg 4, 53121 Bonn, Germany. ${ }^{4}$ Present Address: Deutsches Krebsforschungszentrum (DKFZ), Klinische Kooperationseinheit Pädiatrische Onkologie (G340) and Pädiatrie III, Zentrum für Kinder- und Jugendmedizin, Im Neuenheimer Feld 280, 69120 Heidelberg, Germany.}

\section{Acknowledgements}

The authors thank Kristoffer Riecken and Boris Fehse (Forschungsabteilung Zell- und Gentherapie, Interdisziplinäre Klinik und Poliklinik für Stammzelltransplantation, Universitätsklinikum Hamburg-Eppendorf, Germany) for the provision of the LeGO vectors. The work was supported by the Hilfe für krebskranke Kinder Frankfurt e.V., the Frankfurter Stiftung für krebskranke Kinder, and the Kent Cancer Trust.

\section{Compliance with ethical guidelines}

\section{Competing interests}

The authors declare that they have no competing interests.

Received: 18 July 2015 Accepted: 31 August 2015

Published online: 28 September 2015

\section{References}

1. Glover DM, Leibowitz MH, McLean DA, Parry H. Mutations in aurora prevent centrosome separation leading to the formation of monopolar spindles. Cell. 1995;81:95-105.

2. Kollareddy M, Zheleva D, Dzubak P, Brahmkshatriya PS, Lepsik M, et al. Aurora kinase inhibitors: progress towards the clinic. Investig New Drugs. 2012;30:2411-32.
3. Barr PM, Li H, Spier C, Mahadevan D, LeBlanc M, UI Haq M, et al. Phase Il intergroup trial of alisertib in relapsed and refractory peripheral T-cell lymphoma and transformed mycosis fungoides: SWOG 1108. J Clin Oncol. 2015:33:2399-404

4. Melichar B, Adenis A, Lockhart AC, Bennouna J, Dees EC, Kayaleh O, et al. Safety and activity of alisertib, an investigational aurora kinase A inhibitor, in patients with breast cancer, small-cell lung cancer, non-small-cell lung cancer, head and neck squamous-cell carcinoma, and gastrooesophageal adenocarcinoma: a five-arm phase 2 study. Lancet Oncol. 2015;16:395-405.

5. Sells TB, Chau R, Ecsedy JA, Gershman RE, Hoar K, Huck J, et al. MLN8054 and alisertib (MLN8237): discovery of selective oral aurora A inhibitors. ACS Med Chem Lett. 2015;6:630-4

6. Shang X, Burlingame SM, Okcu MF, Ge N, Russell HV, Egler RA, et al. Aurora $A$ is a negative prognostic factor and a new therapeutic target in human neuroblastoma. Mol Cancer Ther. 2009;8:2461-9.

7. Morozova O, Vojvodic M, Grinshtein N, Hansford LM, Blakely KM, Malova A, et al. System-level analysis of neuroblastoma tumor-initiating cells implicates AURKB as a novel drug target for neuroblastoma. Clin Cancer Res. 2010;16:4572-82

8. Maris JM, Morton CL, Gorlick R, Kolb EA, Lock R, Carol H, et al. Initial testing of the aurora kinase A inhibitor MLN8237 by the Pediatric Preclinical Testing Program (PPTP). Pediatr Blood Cancer. 2010;55:26-34.

9. Carol H, Boehm I, Reynolds CP, Kang MH, Maris JM, Morton CL, et al. Efficacy and pharmacokinetic/pharmacodynamic evaluation of the aurora kinase A inhibitor MLN8237 against preclinical models of pediatric cancer. Cancer Chemother Pharmacol. 2011;68:1291-304.

10. Faisal A, Vaughan L, Bavetsias V, Sun C, Atrash B, Avery S, et al. The aurora kinase inhibitor CCT137690 downregulates MYCN and sensitizes MYCNamplified neuroblastoma in vivo. Mol Cancer Ther. 2011;10:2115-23.

11. Brockmann M, Poon E, Berry T, Carstensen A, Deubzer HE, Rycak L, et al. Small molecule inhibitors of aurora-a induce proteasomal degradation of $\mathrm{N}$-myc in childhood neuroblastoma. Cancer Cell. 2013:24:75-89.

12. Horwacik I, Durbas M, Boratyn E, Węgrzyn P, Rokita H. Targeting GD2 ganglioside and aurora A kinase as a dual strategy leading to cell death in cultures of human neuroblastoma cells. Cancer Lett. 2013;341:248-64.

13. Muscal JA, Scorsone KA, Zhang L, Ecsedy JA, Berg SL. Additive effects of vorinostat and MLN8237 in pediatric leukemia, medulloblastoma, and neuroblastoma cell lines. Investig New Drugs. 2013;31:39-45.

14. Romain C, Paul P, Kim KW, Lee S, Qiao J, Chung DH, et al. Targeting Aurora kinase-A downregulates cell proliferation and angiogenesis in neuroblastoma. J Pediatr Surg. 2014;49:159-65.

15. Michaelis M, Selt F, Rothweiler F, Löschmann N, Nüsse B, Dirks WG, et al. Aurora kinases as targets in drug-resistant neuroblastoma cells. PLoS One. 2014;9:e108758.

16. Morgenstern DA, Baruchel S, Irwin MS. Current and future strategies for relapsed neuroblastoma: challenges on the road to precision therapy. J Pediatr Hematol Oncol. 2013;35:337-47.

17. Park JR, Bagatell R, London WB, Maris JM, Cohn SL, Mattay KK, et al. Children's Oncology Group's 2013 blueprint for research: neuroblastoma. Pediatr Blood Cancer. 2013:60:985-93.

18. Carr-Wilkinson J, O'Toole K, Wood KM, Challen CC, Baker AG, Board JR, et al. High frequency of p53/MDM2/p14ARF pathway abnormalities in relapsed neuroblastoma. Clin Cancer Res. 2010;16:1108-18.

19. Chen L, Tweddle DA. p53, SKP2, and DKK3 as MYCN target genes and their potential therapeutic significance. Front Oncol. 2012;2:173.

20. Guo J, Anderson MG, Tapang P, Palma JP, Rodriguez LE, Niquette A, et al. Identification of genes that confer tumor cell resistance to the aurora B kinase inhibitor, AZD1 152. Pharmacogenom J. 2009;9:90-102.

21. Tavanti E, Sero V, Vella S, Fanelli M, Michelacci F, Landuzzi L, et al. Preclinical validation of aurora kinases-targeting drugs in osteosarcoma. $\mathrm{Br}$ J Cancer. 2013:109:2607-18.

22. Stacy AE, Jansson PJ, Richardson DR. Molecular pharmacology of ABCG2 and its role in chemoresistance. Mol Pharmacol. 2013:84:655-69.

23. Jekerle V, Klinkhammer W, Reilly RM, Piquette-Miller M, Wiese M. Novel tetrahydroisoquinoline-ethyl-phenylamine based multidrug resistance inhibitors with broad-spectrum modulating properties. Cancer Chemother Pharmacol. 2007;59:61-9.

24. Kotchetkov R, Driever PH, Cinatl J, Michaelis M, Karaskova J, Blaheta R, et al. Increased malignant behavior in neuroblastoma cells with acquired 
multi-drug resistance does not depend on P-gp expression. Int J Oncol. 2005;27:1029-37.

25. Rothweiler F, Michaelis M, Brauer P, Otte J, Weber K, Fehse B, et al. Anticancer effects of the nitric oxide-modified saquinavir derivative saquinavirNO against multidrug-resistant cancer cells. Neoplasia. 2010;12:1023-30.

26. Michaelis M, Rothweiler F, Nerreter T, Sharifi M, Ghafourian T, Cinatl J Jr. Karanjin interferes with $A B C B 1, A B C C 1$, and $A B C G 2$. J Pharm Pharm SCi. 2014;17:92-105

27. Weber K, Bartsch U, Stocking C, Fehse B. A multicolor panel of novel lentiviral "gene ontology" (LeGO) vectors for functional gene analysis. Mol Ther. 2008;16:698-706.

28. Weber K, Mock U, Petrowitz B, Bartsch U, Fehse B. Lentiviral gene ontology (LeGO) vectors equipped with novel drug-selectable fluorescent proteins: new building blocks for cell marking and multi-gene analysis. Gene Ther. 2010;17:511-20.

29. Michaelis M, Matousek J, Vogel JU, Slavik T, Langer K, Cinatl J, et al. Bovine seminal ribonuclease attached to nanoparticles made of polylactic acid kills leukemia and lymphoma cell lines in vitro. Anticancer Drugs. 2000;11:369-76.

30. leiri I. Functional significance of genetic polymorphisms in P-glycoprotein (MDR1, ABCB1) and breast cancer resistance protein (BCRP, ABCG2). Drug Metab Pharmacokinet. 2012;27:85-105.
Submit your next manuscript to BioMed Central and take full advantage of:

- Convenient online submission

- Thorough peer review

- No space constraints or color figure charges

- Immediate publication on acceptance

- Inclusion in PubMed, CAS, Scopus and Google Scholar

- Research which is freely available for redistribution

Submit your manuscript at www.biomedcentral.com/submit 